\title{
Validating Real-Time Quantitative RT-PCR Amplicons From Linearly Amplified Primitive Hematopoietic Progenitor mRNA
}

\author{
Matthew S. Nelson ${ }^{1}$, Yonghong Xie ${ }^{1}$, Anil C. Asrani ${ }^{2}$, Kalpna Gupta ${ }^{3}$ and Pankaj Gupta*,1,3 \\ ${ }^{1}$ Hematology-Oncology Section, VA Medical Center, Minneapolis, MN 55417; Departments of ${ }^{2}$ Diagnostic \& Biological \\ Sciences and ${ }^{3}$ Hematology/Oncology/Transplantation Division, Department of Medicine; University of Minnesota, \\ Minneapolis, MN 55455, USA
}

\begin{abstract}
Objective. Low gene expression in rare cell subpopulations can make it difficult to identify transcripts using real time quantitative RT-PCR (qRT-PCR). Transcript number can be increased using linear amplification, but this technique amplifies the 3' end of mRNA, imposing severe limitations on qRT-PCR primer design. Artifacts such as primer-dimers, introduced by these limitations, may interfere with accurate identification of genuine qRT-PCR amplicons. We explore the use of a sequential strategy to distinguish genuine amplicons from primer dimers.

Methods. Cellular mRNA from CD34 ${ }^{+} / \mathrm{CD} 38^{-} /$lin $^{-}$human umbilical cord blood (UCB) progenitors was linearly amplified using the RiboAmp Kit. qRT-PCR was performed using 3' primers to assay bone morphogenetic protein (BMP) signaling cascade expression. PCR products were analyzed for their size, dissociation temperature and sequence specificity.

Results. We found that 18/31 (58\%) of primer pairs formed artifactual products due to primer dimers. Importantly, primer dimer prediction scores did not correlate well with the presence or absence of primer dimer formation under actual experimental conditions. Using a sequential strategy to generate a Primer Profile for each primer set, described here in detail for one gene (BMP-2) that was variably expressed under different culture conditions, we were able to distinguish genuine amplicons from primer dimers. Similar differences were seen between genuine amplicons and primer dimers for several other genes.
\end{abstract}

Conclusions. These findings demonstrate the need for using such a strategy to detect false positive qRT-PCR results, particularly when using linear amplification of mRNA and SYBR Green.

\section{INTRODUCTION}

Real-time quantitative reverse transcription polymerase chain reaction (qRT-PCR) is a powerful tool for directly measuring gene expression and for validating the results of microarray experiments [1, 2]. Enrichment for the most primitive hematopoietic progenitors yields small numbers of cells and thus small amounts of mRNA that may require linear amplification $[3,4]$ for qRT-PCR assays.

Since linear amplification amplifies the 3' end of mRNA, two sequential rounds of amplification yield amplified RNA (aRNA) that ranges from 200-800 bases in length [5]. A linear relationship between the amounts of original mRNA and the amplified product is thus only assured within the first few hundred bases from the 3' end. Therefore, in order for real-time quantitative RT-PCR to provide an accurate measure of the abundance of the original mRNA, primers need to be designed within these first few hundred bases from the 3' end. This short length of 3' aRNA limits the amount of sequence available for use when designing primers, [5] necessitating the use of primers that may be suboptimal and form primer dimers. When using SYBR Green for detection of double-stranded products in qRT-

*Address correspondence to this author at the Hematology/Oncology Section (111E), VA Medical Center, One Veterans Drive, Minneapolis, MN 55417, USA; Tel: (612)-467-4135; Fax: (612)-725-2149;

E-mail: gupta013@umn.edu
PCR, primer dimers can appear as amplified products that can be mistaken for genuine amplicons $[1,6,7]$. Validation of the results is therefore essential.

Using linear amplification and qRT-PCR to assay expression of 31 genes of the bone morphogenetic protein (BMP) signaling cascade in $\mathrm{CD} 34^{+} / \mathrm{CD} 38^{-} / \mathrm{lin}^{-}$human umbilical cord blood (UCB) progenitors, we found that $18 / 31(58 \%)$ of primer pairs formed false positive products (primer dimers). Of these, 8 formed only primer dimers and 10 formed both primer dimers and genuine amplicons. To distinguish genuine target amplicons from primer dimers, we therefore used a five-step sequential strategy to initially generate a Primer Profile for each primer set: (1) water blank and positive control (2) dissociation curve analysis (3) serial dilution of sample RNA (4) gel electrophoresis and (5) product sequencing, which we describe for one gene (BMP2). For each primer set, the Primer Profile allowed us to determine the melting temperatures of genuine amplicons and of primer dimers. In subsequent experiments, we were then able to reliably deduce which products were genuine amplicons.

\section{MATERIALS AND METHODOLOGY}

\section{Purification and Processing of RNA from UCB Cells}

$\mathrm{CD}^{+} / \mathrm{CD}^{-} 38^{-} / \mathrm{lin}^{-}$human UCB cells were cultured for 18 $\mathrm{h}$ in conditions that induced BMP-2 expression (BMP-2 $2^{+}$; long-term bone marrow culture [LTBMC] medium with 10 
$\mathrm{ng} / \mathrm{ml}$ each of Flt3-ligand, thrombopoietin and BMP-4 and 5 $\mathrm{mg} / \mathrm{ml} \mathrm{N}$-desulfated heparin) or those that did not induce BMP-2 expression (BMP-2 ; LTBMC medium with 10 $\mathrm{ng} / \mathrm{ml}$ Sonic hedgehog) [8, 9]. Total RNA from 5,000 20,000 cells was isolated using the PicoPure RNA Isolation Kit (Arcturus, Mountain View, CA) and used in 2 rounds of linear amplification of mRNA using the RiboAmp Kit (Arcturus) per manufacturers' instruction. The quantity and quality of aRNA was determined using an Agilent 2100 Bioanalyzer (Agilent, Palo Alto, CA), and 500 ng used in a cDNA synthesis reaction using Superscript III First Strand Synthesis System for RT-PCR with random hexamers (Invitrogen, Carlsbad, CA) per manufacturers' instructions.

\section{Primer Design}

Primers were designed online using the Primer 3 website (http://frodo.wi.mit.edu/cgi-bin/primer3/primer3_www_slow.cgi) [1]. All primers were required to be in the 3 ' region of the aRNA and to be 17-27 bases in length, with an optimum size of 20 bases. The majority of primers were designed from within the first 300 bases from the 3' end, with only one primer designed from within a sequence longer than 350 bases (that for Nodal, up to 368 bases from the 3 ' end). The Max poly $\mathrm{x}$ was set to 3 bases and the GC clamp was set to 2. $\mathrm{T}_{\mathrm{m}}$ was $57^{\circ}-63^{\circ} \mathrm{C}$ with an optimum $\mathrm{T}_{\mathrm{m}}$ of $60^{\circ} \mathrm{C}$. PCR products were predicted to be $50-150 \mathrm{bp}$, with an optimum size of $100 \mathrm{bp}$. Whenever possible, primers were designed to flank an intron in order to detect possible DNA contamination [1] on subsequent gel electrophoresis of amplified products. All primers and predicted amplicons were checked using BLAST (NCBI) to ensure that there was no sequence similarity to other non-target sequences. The top-ranked primer sets (Table 1) were ordered from Sigma Genosys (The Woodlands, TX).

\section{qRT-PCR}

A qRT-PCR reaction $\left(95^{\circ} \mathrm{C}, 15 \mathrm{~s}\right.$ [denaturation]; $60{ }^{\circ} \mathrm{C}$, $40 \mathrm{~s}$ [annealing/elongation]) was performed on an ABI 7900 HT Sequence Detection System (ABI, Foster City, CA), using specific primers and SYBR Green PCR mix (Invitrogen). We confirmed that the isolated $\mathrm{CD} 34^{+} / \mathrm{CD} 38^{-}$ /lin ${ }^{-}$UCB cells expressed high levels of HoxB4, $\beta$-catenin and Notch (not shown), indicating that this cell population was enriched for primitive hematopoietic progenitors.

\section{Agarose Gel Electrophoresis and Sequencing of qRT- PCR Products}

qRT-PCR products were separated on a $2 \%$ agarose gel, visualized by ethidium bromide, and sequenced at the DNA Sequencing and Analysis Center at the University of Minnesota using an ABI 3730xl DNA Analyzer and ABI BigDye Terminator 3.1 Chemistry. Sequences were verified by a BLAST search using the NCBI website.

\section{Literature Search for qRT PCR Determination of Gene Expression in Stem Cells}

A PubMed search for the following key words: quantitative real time RT-PCR, stem cell and expression returned 104 citations. Of 89 papers available online as full text, 44 reported using SYBR Green and 45 reported using TaqMan PCR. Papers were scored for specifically reporting the use of the following controls and validation techniques:
Table 1. Primer Sequences Used

\begin{tabular}{|c|c|c|}
\hline $\begin{array}{l}\text { Transcript } \\
\text { Examined }\end{array}$ & Left Primer (5'- 3') & Right Primer(5'- 3') \\
\hline$\beta$-actin* & tcccccaacttgagatgtatgaag & aactggtctcaagtcagtgtacagg \\
\hline BMP-2 & atcatgccattgttcagacg & ctcgtcaaggtacagcatcg \\
\hline BMP-3 & ttgaagccatcaaatcatgc & ggtacacagcaaggctcagg \\
\hline BMP-4 & acaccacacacacacgttcc & ggtcaaggtgaatgtttaggg \\
\hline BMP-5 & tgctccaaccaaattaaatgc & cagccacatgagcgtactacc \\
\hline BMP-6 & ccacagggttagaaccaacg & tctgagcctcactttctacagg \\
\hline BMP-7 & acggactcgtttccagagg & ttcetttcgcacagacacc \\
\hline BMP receptor 1B & aagccttgaacatcgtcctg & tccttctgggagcttctctg \\
\hline BMP receptor 2 & taagctgtctgaagecttgc & tcagctttcatagtggcatcc \\
\hline Activin receptor 1 & ccattacccacgtgacacc & cagagtttaaatgcacgtaatgg \\
\hline $\begin{array}{l}\text { Activin like } \\
\text { receptor } 1\end{array}$ & gaagaaggtggtgtgtgtgg & tctgagctaggectgagagg \\
\hline Activin receptor 2 & gcatcttgattgaacatcatttacc & gggatatgggttgagactgc \\
\hline Activin receptor $2 \mathrm{~B}$ & tggtgcacaagaagatgagg & atcatggtcccagcactcc \\
\hline Nodal & agagtccgtcaatctcattgc & cagagaaccactccagtgagc \\
\hline GDF-5 & atttgtgcctggtgacttcc & agccctctcctcttctctcc \\
\hline GDF-11 & agagctccetccactcttcc & gcctccctctgttgtattgc \\
\hline SMAD-1 & aaattgcctacatgtttcaatacc & aaagcctatttctgtactgtaaactcc \\
\hline SMAD-5 & tcgaagaggattgtaatcatgg & cctacagtgcagccactagc \\
\hline ID-1 & gaggaattacgtgctctgtgg & caggctggatgcagttaagg \\
\hline ID-3 & ggctgctctccaaactatgc & gggcaacagaacctttctcc \\
\hline HOX B4 & $\begin{array}{c}\text { tccagagttgtacagcagaag } \\
\mathrm{c}\end{array}$ & agagggctctgcaacatcc \\
\hline Notch 1 & gtgagggacgtcagacttgg & gctgccetgaggagtgc \\
\hline CTNN $\beta 1$ & tttgggatatgtatgggtaggg & cagcaggttacaacaactttgg \\
\hline
\end{tabular}

*The $\beta$-actin primers are those that are recommended in the RiboAmp Handbook. GDF: growth/differentiation factor; HOX: homeobox; ID: inhibitor of DNA binding; SMAD: mothers against DPP homolog.

(1) product was run on a gel to determine if its size was correct (2) product was purified and sequenced to verify if it was the target product (3) dissociation curves were used to determine if multiple products were amplified (4) appropriate negative controls were used and (5) serial dilution of the sample RNA was performed to check for linearity of quantification of the specific product.

\section{RESULTS}

\section{qRT-PCR Product Analysis for BMP-2}

BMP-2 primers generated the correct amplicon from BMP- $2^{+}$cDNA (Fig. 1A) and from positive control cDNA (SaOS-2 human osteosarcoma cells; not shown). We found that BMP-2 is variably expressed in UCB progenitors under different culture conditions. Unexpectedly, a product was 
also generated from BMP-2-negative cDNA (Fig. 1B) and from the water blank (not shown). Although the genuine and the false positive qRT-PCR products appeared similar on amplification $\left(\mathrm{DR}_{\mathrm{n}}\right.$ vs cycle) plots (Fig. 1A and 1B), analysis of the derivative dissociation curves showed that their dissociation temperatures $\left(81^{\circ} \mathrm{C}\right.$ for the genuine amplicon and $77^{\circ} \mathrm{C}$ for the primer dimer) and appearance of the peaks were distinct (Fig. 1C and 1D). Since the false positive appeared in the water blank and the negative control, we assumed it was a primer dimer rather than a splice variant. Two-fold serial dilutions of BMP- $2^{+}$cDNA and SaOS-2 cDNA yielded products with the expected one-cycle increase in cycle threshold (CT) for each dilution (Fig. 1E and 1F), whereas primer dimer product CTs did not change with serial dilution of BMP-2 cDNA (Fig. 1G). Importantly, primer dimers also formed at higher dilutions $(\geq 1: 16)$ of BMP- $2^{+}$cDNA (Fig. 1E), possibly because of low target mRNA abundance.

\section{Gel Electrophoresis and Sequence Analysis}

Agarose gel electrophoresis of qRT-PCR products from the BMP- $2^{+}$cDNA showed the expected amplicon size (107 bp) whereas the primer dimer product was smaller (approximately $50 \mathrm{bp}$; insets in Fig. 2A and 2B). Sequence analysis of qRT-PCR products confirmed that the $107 \mathrm{bp}$ product was identical with the 3' end of human BMP-2 (Fig. 2C). The primer dimer product did not yield any sequence. Similar differences were seen between genuine amplicons and primer dimers for several other genes examined in UCB cells and/or SaOS-2 cells, including BMP-3, $-4,-5$ and -7 , GDF-5, BMP receptors BMPR-IB and ACVR-L1, ID-1, Notch and Nodal (not shown).

\section{Primer Profiles}

A BMP-2 Primer Profile for identifying the genuine amplicon and the corresponding primer dimer was created from these data, including the melting temperatures, amplicon sizes and verification of amplicon sequence. Similar Primer Profiles were created for each primer set for other transcripts examined (Table 2). In subsequent experiments, the Primer Profile was used to reliably deduce which products were genuine amplicons by inspection of their melting temperatures [10]. After initial verification of products and generation of a primer profile, correct amplicons can thus subsequently be identified by dissociation curve appearance and temperature, without the need for sequencing each product.

Table 2 also shows the predicted likelihood of annealing or formation of dimers from the individual primers and primer pairs. The requirement for using primer pairs that would amplify from within the first few hundred bases from the 3' end of each mRNA resulted in even the best primer pairs (chosen and used herein) having at least some likelihood of primer dimer formation. However, whether or not primer dimers actually form during a PCR reaction depends not only on the sequences of the primers chosen, but on additional factors such as the dissociation temperatures of the products and abundance of the desired target aRNA. For these reasons, there was no obvious correlation between the complementarity scores and the actual formation of primer dimers in our experiments (Fig. 3). The implication of this finding is that complementarity scores alone should not be
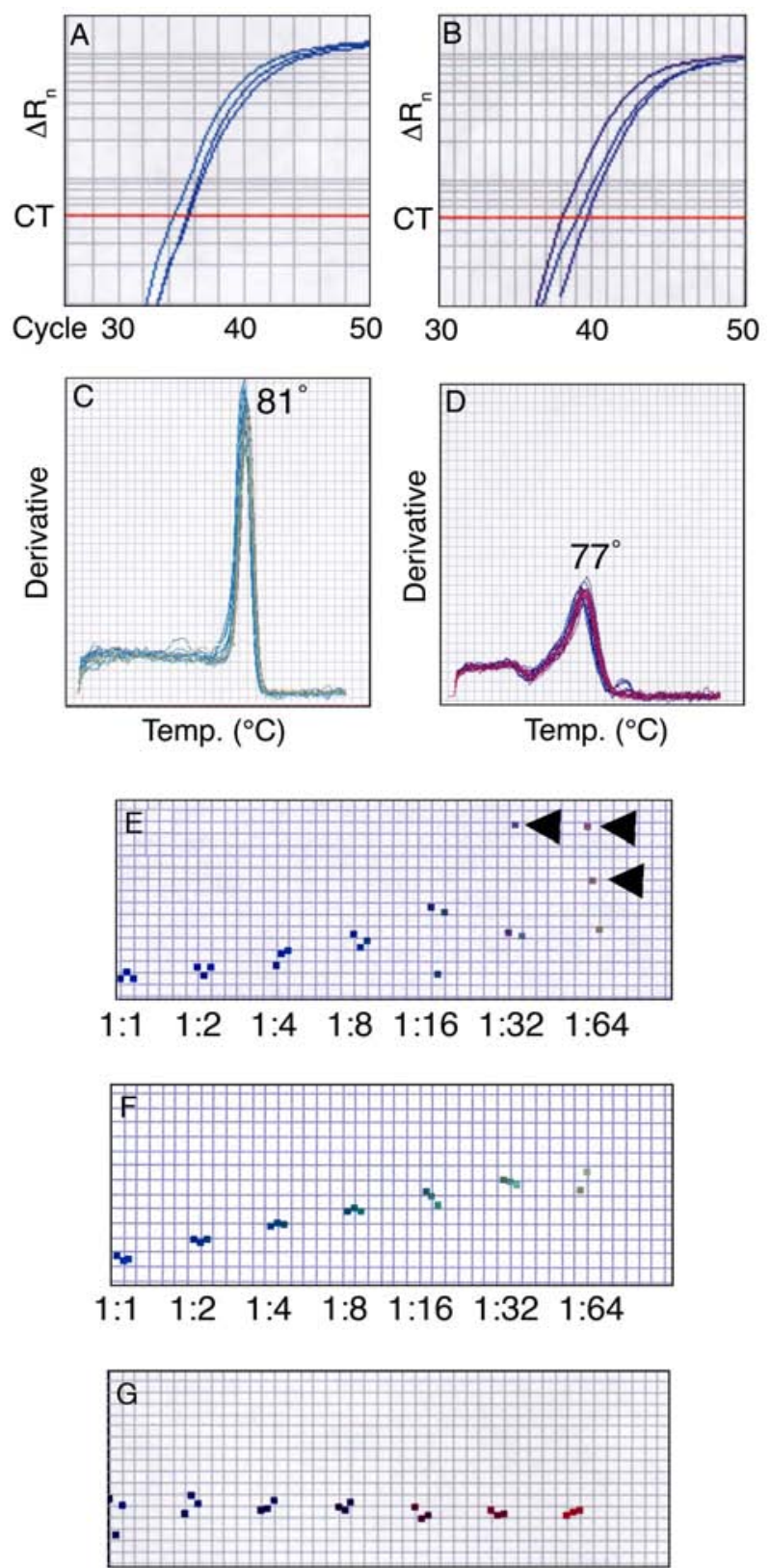

$\begin{array}{lllllll}1: 1 & 1: 2 & 1: 4 & 1: 8 & 1: 16 & 1: 32 & 1: 64\end{array}$

Fig. (1). Analysis of qRT-PCR reaction and products using BMP-2 primers.

$\mathrm{CD}^{2} 4^{+} / \mathrm{CD} 38^{-} / \mathrm{lin}^{-}$human $\mathrm{UCB}$ cells were cultured in conditions that induced BMP-2 expression (BMP- $2^{+}$: panels A, C and E) or those that did not induce BMP-2 expression (BMP-2: panels B, D and $G$ ). (A) shows the $D_{n}$ vs cycle number plot for triplicates of the genuine BMP-2 amplicon and (B) for the primer dimer. (C) shows the dissociation curves for replicates of the genuine BMP-2 amplicon and (D) for the primer dimer. (E) shows the CT plots of 2 -fold serial dilutions of cDNA from BMP-2 ${ }^{+} \mathrm{UCB}$ cells, $(\mathrm{F})$ from the SaOS-2 cell line (positive control), and (G) from BMP- $2^{-}$UCB cells. Arrowheads in (E) indicate primer dimers formed at higher dilutions of BMP- $2^{+}$cDNA.

used to select or reject otherwise suitable primers, and that all reactions should, at least initially, be validated as described here. 

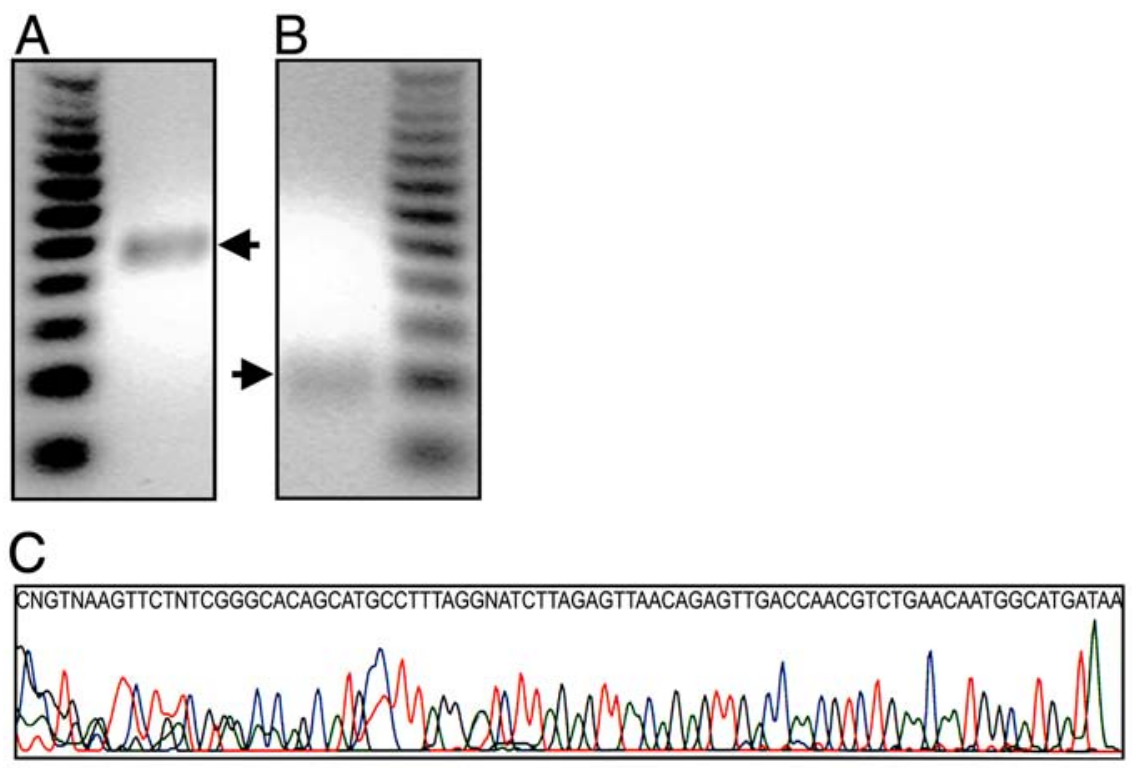

Fig. (2). Electrophoretic separation of genuine amplicon from primer dimer, and sequence analysis of genuine amplicon of BMP-2.

aRNA was subjected to qRT-PCR, electrophoresis and sequencing of amplicons as described in Methods. (A) shows the sizes of the genuine amplicon and (B) of the primer dimer (arrowheads). (C) shows the sequence chromatogram of the genuine amplicon from BMP- ${ }^{+} \mathrm{UCB}$ cells.

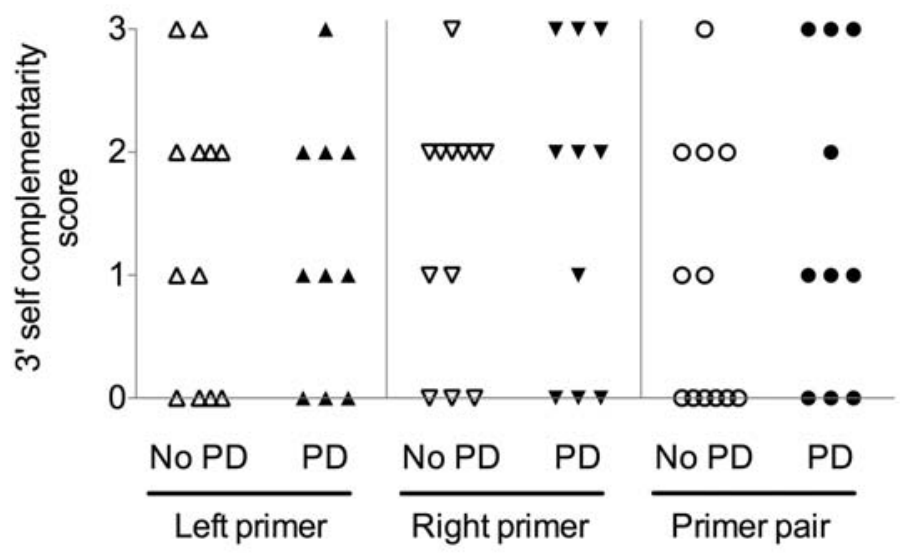

Fig. (3). Relationship between 3' self complementarity scores for primers and the actual formation of primer dimers on qRT-PCR.

The 3' anchored global alignment scores for complementarity (Table 1), reflecting the likelihood of PCR-priming primer dimers, were plotted separately for reactions where primer dimers (PD) actually formed and reactions where primer dimers did not form (No PD) during qRT-PCR. Differences between the respective groups (No PD vs PD) were not statistically significant.

\section{Reporting of Validation Techniques and Controls in the Recent Literature}

Of 89 papers using qRT-PCR for determination of gene expression by various types of stem cell populations, only a variable proportion reported specifically on the use of validation strategies and controls (Table 3). The use of negative controls was reported by only $23 \%$ and $33 \%$ and amplicon sequencing by only $7 \%$ and $4 \%$ of papers where SYBR Green and TaqMan were used respectively. Confirmation of amplicon size was reported by $<40 \%$ of SYBR Green users.

\section{DISCUSSION}

Primer dimer formation may compete with genuine product amplification when target transcript levels are low (as demonstrated by serial dilutions of cDNA in Fig. 1E). In a RT- (lacking cDNA) or water blank reaction that produces a primer dimer product, the CT will not increase with dilution of the template because the concentration of the primers is equal in each dilution and they themselves are the template for the artifact seen. Further, some primer sets (BMP-3, -4 and -7; BMP Receptor-1A; ACVR-L1; Inhibin B; Patched-2 and ID-1 and -2) generated multiple primer dimer peaks with different melting temperatures on the dissociation curves. Since a primer dimer can have a normallooking amplification plot and a single dissociation peak, careful comparison of each qRT-PCR reaction with its Primer Profile is particularly important to identify these artifacts. As shown here, primer dimers appear as products when using SYBR Green since this reagent binds to any double stranded DNA [1, 6, 7]. Initially, analysis by gel 
Table 2. Primer Profiles

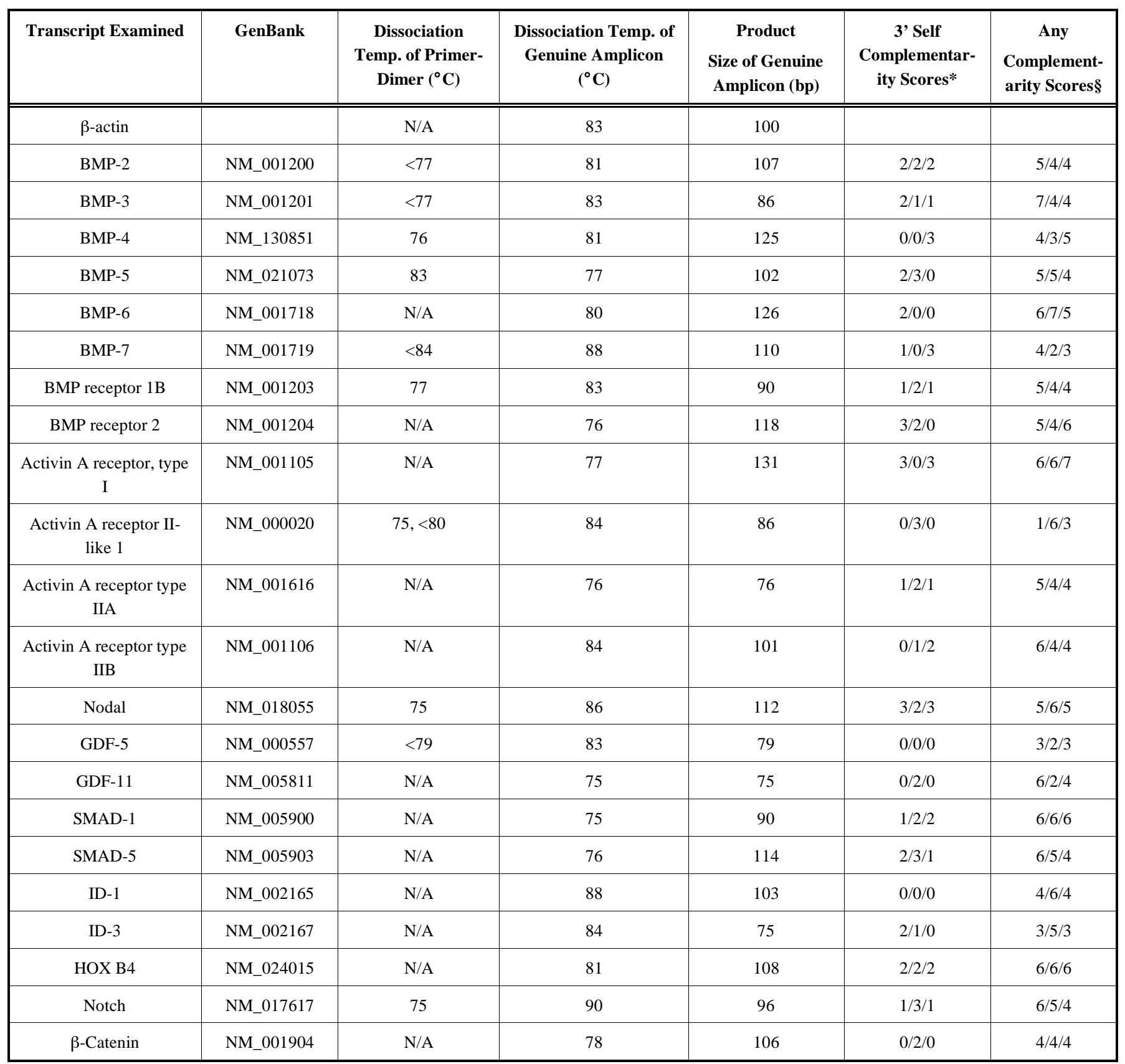

The primer profiles for selected transcripts expressed by UCB cells are shown and include GenBank identification of the sequence used to design primers, the dissociation temperatures of the primer dimers and genuine amplicons, the predicted product sizes of the genuine amplicons and scores indicating the predicted likelihood of forming primerdimers and/or secondary structures. $\beta$-actin primer sequences were provided in the RiboAmp manual from Arcturus. The sequences of all the genuine amplicons shown above (including $\beta$-actin) were verified.

* $\S$ The table also shows the predicted likelihood of the selected primers to anneal to themselves or each other and/or form primer dimers in PCR, calculated as described at http://fokker.wi.mit.edu/primer3/input-help-040.htm\#PRIMER_SELF_ANY

Briefly:

* 3' self-complementarity: This 3'-anchored global alignment score predicts the likelihood of PCR-priming primer dimers. Higher scores indicate increasing likelihood of alignment, while a score of 0.0 indicates no likelihood of alignment.

$\S$ Any complementarity: This local alignment score predicts the likelihood of single primers or primer pairs to anneal to each other without necessarily causing self-priming in PCR In both the 3'Self-Complementarity and Any Complementarity columns, the numbers shown for each primer set indicate the scores for the likelihood of self-complementarity of the left primer/right primer/primer pair, respectively.

GDF: growth/differentiation factor; HOX: homeobox; ID: inhibitor of DNA binding; SMAD: mothers against DPP homolog; N/A: not applicable, since a primer dimer did not form.

electrophoresis and sequencing are essential to ensure that the primer profile and amplicon are correct. If Taqman probes designed to bind specifically to the amplicon sequence between the primers are used instead of SYBR Green, primer dimers will still be formed but not visualized $[1,6,11]$. However, when examining several transcripts, the cost of generating Taqman probes for each one may outweigh this potential advantage of Taqman over SYBR Green [1]. The use of SYBR Green may require more screening initially, but is substantially less expensive when examining large numbers of transcripts. 
Table 3. Reporting of Controls and Validation Results in the Literature

\begin{tabular}{|c|c|c|c|c|c|}
\hline & $\begin{array}{c}\text { Confirmation of } \\
\text { Amplicon Size }\end{array}$ & $\begin{array}{c}\text { Amplicon } \\
\text { Sequencing }\end{array}$ & $\begin{array}{c}\text { Dissociation Curve } \\
\text { Analysis }\end{array}$ & $\begin{array}{c}\text { Negative Controls } \\
\text { Serial Dilutions of } \\
\text { Sample RNA }\end{array}$ \\
\hline \hline Sybr Green & 39 & 7 & 57 & 23 & 18 \\
\hline TaqMan & 2 & 4 & N/A & 33 \\
\hline
\end{tabular}

The percentage of papers that reported performing each specific control or validation technique for both SYBR Green and TaqMan qRT-PCR are shown. N/A: not applicable.

Quantitative real time PCR can be very powerful but can also be misleading. Our findings demonstrate the critical importance of using a strategy to detect false positive SYBR Green qRT-PCR results due to primer dimers when using linear amplification of mRNA from primitive hematopoietic progenitors and other small subpopulations of cells. Inclusion of validation data such as genuine amplicon size and dissociation curve temperature in published papers will assist other laboratories in ensuring the accuracy of their own qRT-PCR reactions when using the same primers.

\section{ACKNOWLEDGEMENTS}

The US Department of Veterans Affairs and NIH R01NS-48606 supported this work.

\section{REFERENCES}

[1] Ginzinger, D.G. Gene quantification using real-time quantitative PCR: an emerging technology hits the mainstream. Exp. Hematol, 2002, 30, 503-12.

[2] Wong, M.L.; Medrano J.F. Real-time PCR for mRNA quantitation. Biotechniques, 2005, 39, 75-85.

[3] Bartolovic, K.; Balabanov, S.; Berner, B; Buhring, H.J.; Komor, M.; Becker, S.; Hoelzer, D.; Kanz, L.; Hofmann, W.K.; Brummendorf, T.H. Clonal heterogeneity in growth kinetics of CD34+CD38- human cord blood cells in vitro is correlated with gene expression pattern and telomere length. Stem Cells, 2005, 23, 946-57.

[4] Van Gelder, R.N.; von Zastrow, M.E.; Yool, A.; Dement, W.C.; Barchas, J.D.; Eberwine, J.H. Amplified RNA synthesized from limited quantities of heterogeneous cDNA. Proc. Natl. Acad. Sci. USA, 1990, 87, 1663-67.

[5] Wilhelm, J.; Muyal, J.P.; Best, J.; Kwapiszewska, G.; Stein, M.M Seeger, W.; Bohle, R.M.; Fink, L. Systematic comparison of T7IVT and SMART-based RNA pre-amplification techniques for DNA microarray experiments. Clin. Chem., 2006, 52, 1161-67.

[6] Ponchel, F.; Toomes, C.; Bransfield, K.; Leong, F.T.; Douglas, S.H.; Field, S.L.; Bell, S.M.; Combaret, V.; Puisieux, A.; Mighell, A.J.; Robinson, P.A.; Inglehearn, C.F.; Isaacs, J.D.; Markham, A.F Real-time PCR based on SYBR-Green I fluorescence: an alternative to the TaqMan assay for relative quantification of gene rearrangements, gene amplifications and micro-gene deletions. BMC Biotechnol., 2003, 3, 18 .

[7] Simpson, D.A.; Feeney, S.; Boyle, C.; Stitt, A.W. Retinal VEGF mRNA measured by SYBR green I fluorescence: a versatile approach to quantitative PCR. Mol. Vis., 2000, 6, 178-83.

[8] Colvin-Wanshura, L.E.; Nelson, M.S.; Stephenson, E.J.; Khan, S.A.; Xie, Y.; Gupta, P. Selective modulation of the BMP signaling pathway has distinct effects on long-term in vitro maintenance of primitive hematopoietic progenitors in human umbilical cord blood. [abstract]. Blood, 2005, 106, 883a.

[9] Gupta, P.; Oegema, T.R.; Brazil, J.J.; Dudek, A.Z.; Slungaard, A.; Verfaillie, C.M. Structurally specific heparan sulfates support primitive human hematopoiesis by formation of a multimolecular stem cells niche. Blood, 1998, 92, 4641-51.

[10] Ririe, K.M.; Rasmussen, R.P.; Wittwer, C.T. Product differentiation by analysis of DNA melting curves during the polymerase chain reaction. Anal. Biochem., 1997, 245, 154-60.

[11] Holland, P.M.; Abramson, R.D.; Watson, R.; Gelfand, D.H. Detection of specific polymerase chain reaction product by utilizing the 5'-3' exonuclease activity of Thermus aquaticus DNA polymerase. Proc. Natl. Acad. Sci. USA, 1991, 88, 7276-80.

Received: October 01, 2008

Revised: October 22, 2008

Accepted: November 10, 2008

(C) Nelson et al.; Licensee Bentham Open.

This is an open access article licensed under the terms of the Creative Commons Attribution Non-Commercial License (http://creativecommons.org/licenses/by-nc/3.0/) which permits unrestricted, non-commercial use, distribution and reproduction in any medium, provided the work is properly cited. 